\title{
Evaluación de la Inteligencia Emocional en la Infancia y la Adolescencia: Una Revisión Sistemática de Instrumentos en Castellano
}

\author{
Emotional Intelligence Assessment in Childhood and Adolescence: A Systematic \\ Review of Measures in Spanish
}

\author{
Christiane Arrivillaga ${ }^{1}$ y Natalio Extremera ${ }^{2}$
}

\begin{abstract}
Resumen
Actualmente se cuenta con instrumentos válidos y fiables para la evaluación de la inteligencia emocional (IE). Sin embargo, el conocimiento sobre la evaluación de la IE en la niñez y la adolescencia en entornos hispanohablantes es más escaso. Con el objetivo de analizar qué medidas están siendo utilizadas con esta población, se llevó a cabo una revisión sistemática de la literatura científica. Se consultaron diez bases de datos, utilizando combinaciones de términos referidos a la inteligencia emocional, infancia, adolescencia, evaluación y adaptación, en español y en inglés. Un total de 68 artículos fueron incluidos en la revisión. Los resultados indicaron que ha sido utilizada una gran variedad de instrumentos de medición de la IE en estas edades. Se discute sobre la adecuación de las medidas en relación al momento evolutivo en el que fueron utilizadas y se presentan algunas reflexiones para guiar el diseño y elección de herramientas de evaluación de la IE en población infanto-juvenil.
\end{abstract}

Palabras clave: inteligencia emocional, revisión sistemática, evaluación, infancia, adolescencia

\begin{abstract}
At present there are reliable and valid measurement instruments for emotional intelligence (EI). However, most research has been conducted on adult English-speaking populations, so knowledge on the assessment of EI in childhood and adolescence in Spanish speaking countries is scarce. The present research aimed at analyzing EI measures in Spanish that are being used with children and adolescents. A systematic review of scientific literature was conducted. Ten databases were consulted, using combinations of the words emotional intelligence, childhood, adolescence, assessment and adaptation, in Spanish and in English. A total of 68 papers were included in the review. Results point to a great variety of measurement instruments used to assess EI at these stages. The adequacy of the measures regarding the parcipants' developmental stage in which the measures were used are discussed and some reflections are given in order to guide the design and choice of EI assessment tools for children and adolescents.
\end{abstract}

Keywords: emotional intelligence, systematic review, assessment, childhood, adolescence

\footnotetext{
${ }^{1}$ Personal investigador. Facultad de Psicología. Universidad de Málaga. Campus de Teatinos s/n, 29071 Málaga, España. Tel.: 952137570. Correo: carrivillagad@uma.es

${ }^{2}$ Doctor en Psicología. Catedrático de Universidad. Facultad de Psicología. Universidad de Málaga. Campus de Teatinos s/n, 29071 Málaga, España. Tel.: 9521323 90. Correo: nextremera@uma.es (Correspondencia) 


\section{Introducción}

El campo de investigación de la inteligencia emocional (IE) se encuentra en su tercera década de desarrollo. La IE puede definirse como la habilidad para procesar la información emocional y la capacidad para utilizar las emociones con el objetivo de mejorar el pensamiento (Mayer, Caruso, \& Salovey, 2016). Diversas revisiones y meta-análisis permiten afirmar que es un constructo asociado al bienestar de las personas (ej: Fernández-Berrocal \& Extremera, 2016; Resurrección, Salguero, \& Ruiz-Aranda, 2014; Sánchez-Álvarez, Extremera, \& FernándezBerrocal, 2016). A pesar de los avances alcanzados, la conceptualización de la IE sigue siendo controvertida, ya que existen distintos modelos teóricos (Mayer, Roberts, \& Barsade, 2008).Los enfoques integradores de la IE, también conocidos como modelos de habilidades, se refieren a un conjunto de capacidades cognitivas necesarias para el procesamiento de la información emocional (i.e. Mayer \& Salovey, 1997). Los modelos mixtos, además de algunos aspectos cognitivos, incluyen disposiciones y/o aspectos de personalidad (i.e. Bar-On, 2006; Goleman, 1995; Petrides \& Furnham, 2001). Desde cada modelo teórico se han diseñado diferentes sistemas de evaluación del constructo, llevando a la existencia de una gran cantidad de instrumentos para medir la IE (Extremera \& Fernández-Berrocal, 2007).

Este panorama mencionado corresponde al estado de la cuestión de la IE en población adulta. Sin embargo, la investigación demuestra que en la infancia y la adolescencia existe aún mayor solapamiento teórico, pues a la diversidad de planteamientos anteriores, se suman diversos enfoques y modelos sobre competencias socioemocionales, educación emocional, aprendizaje socioemocional, entre otros (i.e. Bisquerra, 2009; Denham, Wyatt, Bassett, Echeverria, \& Knox, 2009; Humphrey et al., 2011). Consecuentemente, los hallazgos sobre el desarrollo emocional en dichas edades son difíciles de agrupar bajo el mismo rótulo para construir un corpus empírico sólido.

En los contextos hispanohablantes, a las dificultades conceptuales y de medición ya mencionadas, se suman aquellas referidas a la utilización de instrumentos que han sido diseñados para culturas anglosajonas. Con frecuencia se encuentran investigaciones que utilizan cuestionarios que sólo han sido traducidos, sin profundizar sobre los significados culturales de los constructos, por ejemplo, entrevistando a la población diana (International Test Commission, 2017). Utilizar instrumentos no adaptados o validados lleva a una disminución en la calidad de la investigación. Varios autores coinciden en que la falta de instrumentos de evaluación de IE validados en castellano para población infanto-juvenil sigue siendo una limitación para las investigaciones en países hispanohablantes (Cobos-Sánchez, FlujasContreras, \& Gómez-Becerra, 2017; FernándezAngulo, Quintanilla, \& Giménez-Dasi, 2016; Ramírez-Lucas, Ferrando, \& Sáinz, 2015).

El objetivo del presente estudio fue analizar los instrumentos que están siendo utilizados para evaluar la inteligencia emocional en niños y adolescentes de contextos hispanohablantes. Con tal fin, se realizó una revisión sistemática de los artículos empíricos publicados hasta la actualidad.

\section{Metodología}

\section{Diseño}

Se condujo una revisión sistemática de estudios empíricos en los que se realizara una evaluación de la inteligencia emocional en población infantojuvenil en contextos hispano hablantes. Se siguieron las recomendaciones PRISMA en el proceso de revisión (Moher, Liberati, Tetzlaff, \& Altman, 2010), las cuales incluyen los ítems que deben ser reportados a la hora de conducir una revisión sistemática de literatura.

\section{Búsqueda inicial}

Se consultaron diez bases de datos: Bibliografía de Literatura Española, ERIC, Psycarticles, Psychology Database, PsycInfo, CSIC-ISOC Psicología, SCOPUS, Medline, Scielo y Dialnet. Las primeras cinco se examinaron utilizando el meta buscador ProQuest. Las búsquedas se condujeron entre el 30 de abril y el 10 de junio del 2018. Se utilizaron combinaciones de los términos: inteligencia emocional, infancia, niñez, niños, adolescencia, 
Tabla 1. Términos utilizados en la búsqueda según base de datos y resultados iniciales

\begin{tabular}{|c|c|c|}
\hline Base de datos & Términos de búsqueda & Resultados iniciales \\
\hline ProQuest (Bibliografía de Literatura & ("inteligencia emocional" AND (evaluación OR & \\
\hline Española, ERIC, Psycarticles, & medición) AND (infancia OR niñez OR adolescencia & \\
\hline \multirow[t]{5}{*}{ Psychology Database, PsycInfo). } & OR juventud)) AND peer(yes). & \\
\hline & ("emotional intelligence" AND ab(childhood OR & 222 \\
\hline & child OR adolescence OR adolescent) AND & \\
\hline & ab(assessment OR evaluation OR measurement) & \\
\hline & AND (adaptation OR validation)) AND peer(yes). & \\
\hline \multirow[t]{2}{*}{ CSIC-ISOC Psicología } & “inteligencia emocional” AND (adolescencia OR & \\
\hline & adolescente OR infancia OR niños). & 36 \\
\hline \multirow[t]{5}{*}{ SCOPUS } & $\begin{array}{l}\text { "inteligencia emocional" AND (adolescencia OR } \\
\text { infancia). }\end{array}$ & \\
\hline & "emotional intelligence" AND ab(childhood OR child & 127 \\
\hline & OR adolescence OR adolescent) AND ab(assessment & \\
\hline & OR evaluation OR measurement) AND (adaptation & \\
\hline & OR validation). & \\
\hline \multirow[t]{4}{*}{ Medline } & "emotional intelligence" AND ab(childhood OR child & \\
\hline & OR adolescence OR adolescent) AND ab(assessment & \\
\hline & $\begin{array}{l}\text { OR evaluation OR measurement) AND (adaptation } \\
\text { OR validation). }\end{array}$ & 179 \\
\hline & $\begin{array}{l}\text { ab("emotional intelligence") AND ab(childhood OR } \\
\text { child OR adolescence OR adolescent). }\end{array}$ & \\
\hline \multirow[t]{2}{*}{ Scielo } & ab("inteligencia emocional" AND (adolescencia OR & \\
\hline & $\begin{array}{l}\text { infancia OR niñez)). } \\
\text { ab ("emotional intelligence" AND (adolescent OR } \\
\text { adolescence OR childhood)). }\end{array}$ & 42 \\
\hline Dialnet & $\begin{array}{l}\text { "inteligencia emocional" AND (adolescencia OR } \\
\text { infancia). }\end{array}$ & 166 \\
\hline Total & & 772 \\
\hline
\end{tabular}

adolescente, juventud, evaluación, medición, adaptación y validación, en español y en inglés. En la Tabla 1 se muestran los términos y combinaciones utilizadas en cada base de datos, así como el número de artículos que pasaron a la fase de cribado.

\section{Cribado, criterios de inclusión y exclusión}

Se rastreó en el título, resumen, apartado de participantes y de métodos si se cumplían los siguientes criterios de inclusión: (a) eran investigaciones empíricas, (b) se realizó una evaluación de la inteligencia emocional (más de una dimensión referida exclusivamente a aspectos emocionales), (c) la población era menor de diecisiete años y (d) la evaluación se realizó en castellano. Como criterio de exclusión, se utilizó la no disponibilidad y acceso al texto completo del artículo. En algunos artículos no aparecía la información sobre la edad o las dimensiones de IE evaluadas. Ante tal obstáculo, se mantuvo el artículo para determinar su pertinencia en la siguiente fase.

\section{Análisis del texto completo y selección final de estudios}

En la fase de lectura del texto completo, se analizó en detalle el cumplimiento de los cuatro criterios de inclusión. Por ejemplo, en cuanto a la edad de la muestra de investigación, cuando el límite superior excedía los diecisiete años, pero la media era igual o inferior a este número se procedió a incluir el artículo; de lo contrario se descartaba. No se incluyeron artículos que no hubieran surgido en la búsqueda sistemática (ej: lista de referencias) como input adicional. Los artículos fueron leídos por una investigadora quien registró datos sobre: el año de publicación, las características de la población, rango de edad, técnica de evaluación, modelo de inteligencia emocional, instrumento utilizado, versión, fiabilidad y validez (en los casos en los que fueran reportadas en el estudio).

No fue posible la conducción de un meta análisis formal para estimar el efecto de la inteligencia emocional sobre otros indicadores en la población diana, debido a la heterogeneidad de estudios, la diversidad de constructos evaluados y la falta de datos 
asociados a dichas variables. Por lo tanto, se realizó una revisión sistemática desde una perspectiva cualitativa en la que se presentan los rangos de correlaciones halladas por los instrumentos de evaluación con el propósito de aportar información sobre los indicadores de validez de las medidas.

\section{Resultados}

De acuerdo al procedimiento descrito en el apartado anterior, se cribaron 772 artículos. De los artículos factibles de incluirse por cumplir los criterios establecidos, se encontraron 47 duplicados. Setenta y tres artículos pasaron a la fase de análisis del texto completo (21 de ProQuest, 5 de CSIC-ISOC, 3 de SCOPUS, 5 de Medline, 3 de Scielo y 36 de Dialnet). Se descartaron cinco artículos por no cumplir alguno de los criterios de inclusión (ej: edad media de la muestra era superior a los diecisiete años). Los 68 artículos restantes, los cuáles disponían en su totalidad de acceso al texto completo, fueron incluidos en la revisión.

No se realizó ninguna restricción por año de publicación, tomando, por tanto, todas las publicaciones realizadas desde el origen del constructo hasta la fecha de la revisión en 2018. Tras el análisis de los artículos incluidos en esta revisión, el período en el que fueron publicados los artículos fue del 2003 al 2018. Cincuenta y cinco investigaciones se realizaron con participantes en España y doce en Latinoamérica. Un estudio tenía muestras tanto de México como de España (ver lista de referencias: 25). En cuanto al tamaño muestral, se hallaron desde investigaciones de caso único (49) hasta estudios con muestras de 5754 sujetos (17). A modo de referencia, se estimó que la media de todas las muestras reportadas fue de $676.17(\mathrm{DE}=1470)$ y la mediana fue de 1099.5.

Basándose en la clasificación de Denham, Wyatt, Bassett, Echeverria y Knox (2009), se encontraron dos artículos con muestras en edad preescolar (3 a 6 años; 1, 48), dos con población en edad escolar (7-11 años; 19, 21), tres con adolescentes en la etapa temprana (12-14 años;
$41,63,65)$ y cuatro con adolescentes en etapa media (15-17 años; 10, 26, 34, 49). Trece artículos tenían muestras que abordaban más de un período, pero la mayoría de estudios fueron realizados con población en la adolescencia temprana y media (cuarenta y tres artículos). Todos los sujetos estaban escolarizados.

Los cuestionarios de auto informe o pruebas de rendimiento típico fueron la técnica de evaluación más frecuente (63 artículos), seguida de pruebas de habilidad o rendimiento máximo $(1,4,5,10,40,45,46,47,48)$. En cuanto al modelo teórico de partida, se observó que cuarenta estudios se basaban en el modelo de habilidad de Mayer y Salovey (1997), diecinueve en el enfoque mixto de Bar-On $(2006 ; 3,4,9,13,18,19,21,23,27,48,55,56$, $57,60,62,63,64,66,67)$, cinco se orientaban por un modelo de IE como rasgo (Petrides \& Furnham, 2001; 11, 12, 24, 35, 58), dos hacían alusión al modelo mixto de Goleman (2005; 38, 53), uno a la teoría de conciencia emocional (Rieffe, Oosterveld, Miers, Terwogt, \& Ly, 2008; 59) y en cuatro no se identificó un enfoque predominante $(1,5,26,37)$.

En la Tabla 2 se resumen los resultados de los instrumentos de evaluación de la IE utilizados, las referencias a las versiones que se emplearon, los artículos en los que se utilizó cada instrumento y una síntesis de las propiedades psicométricas reportadas. En la Tabla 3 se presentan los instrumentos de medición utilizados en cada etapa evolutiva, tomando como referencia las categorías propuestas por Denham, Wyatt, Bassett, Echeverria y Knox (2009). Finalmente, en la Tabla 4 se presentan los instrumentos utilizados, junto a la referencia de la primera adaptación en castellano, el país en el que se realizó, el número de ítems del instrumento, qué dimensiones se evalúan y la muestra con la que se realizó la adaptación. 
Tabla 2. Instrumentos de evaluación de la IE utilizados en los artículos revisados

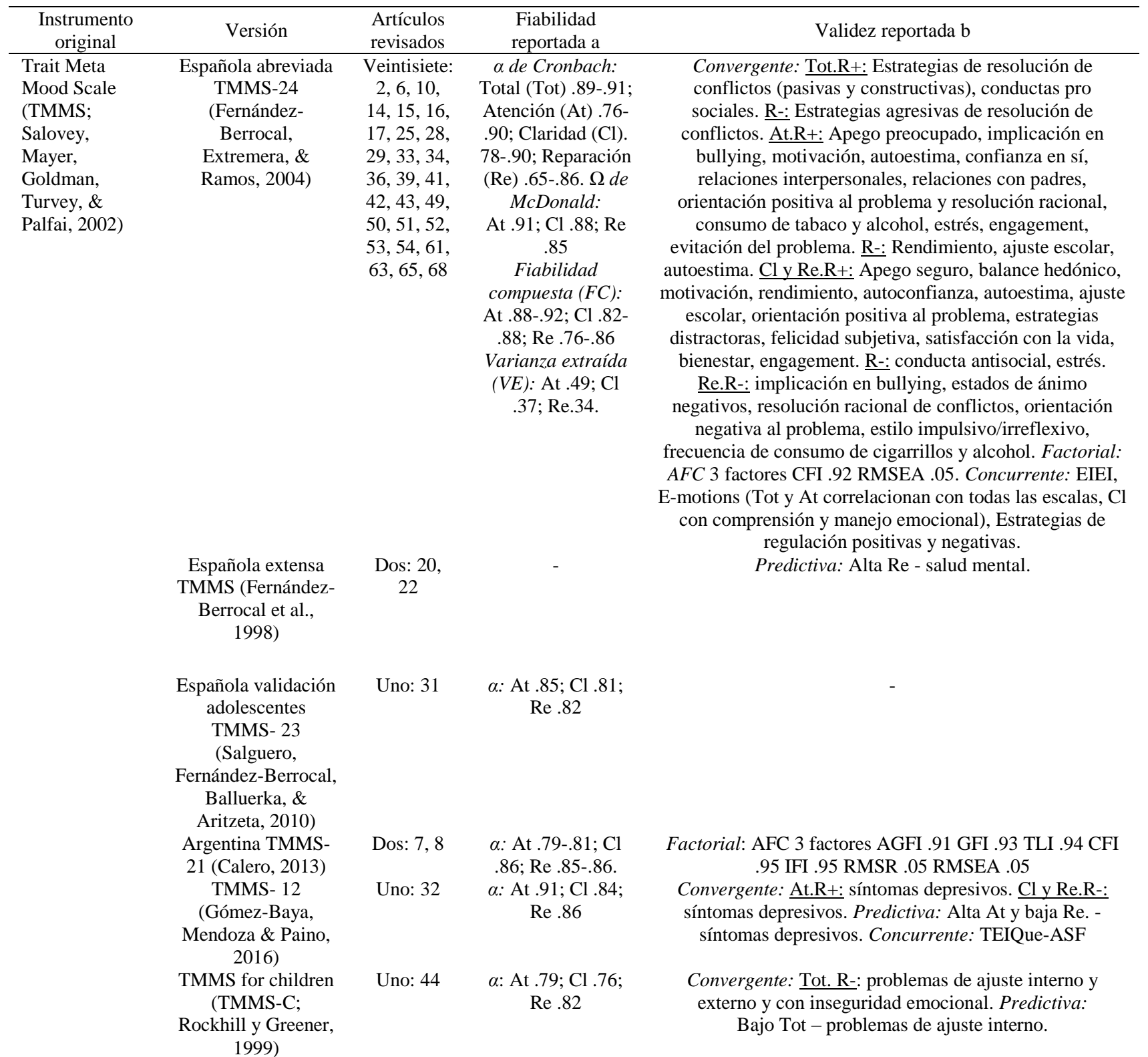

Escala de Inteligencia Emocional en Internet (EIEI; González-Cabrera

Pérez-Sancho, \&

Calvete, 2016)

Emotional Quotient inventoryYouth version (EQi-YV; Bar-On \& Parker, 2000)
Española completa (Ferrándiz, Hernández,

Bermejo, Ferrando, \& Sáinz, 2012)
Uno: 33

$\alpha \cdot$ Tot .89; At .72; Cl .86; Re .89.

Test-retest $r$ : Tot

.77; At .86; Cl .78; Re 69.

Cuatro: $4, \quad \alpha:$ Total (Tot) 89 ; 13, 23, 27 Intrapersonal (Ia) .62- .81;

Interpersonal (Ie) .70- .77; Manejo estrés (Me) .65-

.79; Adaptabilidad (Ad) .77- .78; Humor (Hu).80.84
Convergente: Tot. R-: bullying. Factorial: AFC 3 factores CFI .98 NNFI .98 RMSEA .05. Concurrente: TMMS-24

Convergente: Tot. $\mathrm{R}+$ : relaciones interpersonales, relaciones con padres, autoconfianza, ajuste personal, extroversión, inteligencia. R-: excitabilidad, ansiedad.

Ia.R+: autoestima, autoconfianza, ajuste personal, inteligencia. Ie.R+: relaciones interpersonales, relaciones con padres, ajuste personal. R-: actitud negativa (escuela).

Me.R+: actitud negativa (escuela), búsqueda de sensaciones, conducta atípica, locus de control, somatización, estrés social, ansiedad, depresión, sensación de insuficiencia, desajuste clínico y académico. Ad.R+: relaciones interpersonales, relaciones con padres, autoestima, autoconfianza, ajuste personal, inteligencia. $\underline{\mathrm{R}-}$ : actitud negativa (maestra), depresión, sensación insuficiencia, síntomas emocionales. $\underline{\mathrm{Hu} . \mathrm{R}+\text { : relaciones }}$ interpersonales, relaciones con padres, autoestima, autoconfianza. R-: actitud negativa (escuela y maestra), conducta atípica, locus de control, somatización, estrés social, ansiedad, depresión, sensación de insuficiencia, desajuste clínico y académico, síntomas emocionales. 
Tabla 2. Continuación

Española completa
(Sáinz, Ferrándiz,
Fernández-Vidal, \&
Ferrando, 2014)
Española abreviada
(EQi-YV-S;
Esnaola, Freeman,
Sarasa, Fernández-
Zabala, \& Axpe,
2016)
Mexicana 48 ítems
(Rucalva, Gallegos,
Lorenzo, \& Borges,
2014)

Peruana completa y
abreviada (Ugarriza
\& Pajares, 2005)
Referencia a
instrumento
original: Completa

Española completa

Dos: 21, 57

Uno: 18

Esnaola, Freeman,

Sarasa, Fernández2016)

Mexicana 48 ítems

(Rucalva, Gallegos, 2014)

Dos: 55,
56

$\alpha$ : Tot .90; Ia .77;

Ie .77; Me .82; Ad .84 ;

Emoción positiva .73; Optimismo y autoestima .86

Dos: 3, $62 \quad \alpha$ : Completa Tot

.70- .79; Abreviada

Tot .64- .77

Cinco: $5, \quad \alpha$ : Tot .89- .95; Ia

$63,64,66, \quad .51-.79$; Ie $.70-$

$67 \quad .76 ;$ Me $.57-.82$;

Ad .74- .83; Hu .88

Trait Emotional Intelligence

Questionnaire-

Adolescent

Short Form

(TEIQue-ASF;

Petrides et al., 2006)

Referencia a
trumento origina

Cuatro: 9 ,
19,60

48

Referencia a

instrumento original

EQ-360

Española (Ferrando

et al., 2011)

Uno: 35
$\alpha$ : Tot .80. $\Omega$ : Tot
$.93 V E: .62$

Cinco: 5, $11,12,23$, 24

$\alpha:$ Tot .79- .89

Trait Emotional Intelligence

Questionnaire-

Short Form

(TEIQue-SF;

Petrides, 2012)

Mayer-Salovey-

Caruso

Emotional

Intelligence

Test (MSCEIT;

Mayer,

Salovey, \&

Caruso, 2002)

$\begin{array}{ccc}\text { Referencia a } & \text { Uno: } 58 & \alpha . \text { Tot } .76 ; \\ \text { instrumento original } & & \text { Bienestar .84; } \\ & & \text { Autocontrol .82; } \\ & & \text { Hab. emocionales } \\ & & .85 ; \\ \text { Española } & \text { Uno: } 40 & \text { Hab. sociales } .81 \\ \text { Spearman-Brown: } \\ \text { (Extremera \& } & & \text { Experiencial (Ex) } \\ \text { Fernández-Berrocal, } & & .84 \\ \text { 2002) } & & \text { Estratégica (Est) } \\ & & .63\end{array}$

(Pulido, 2017)
Tres: 45 ,
46,47

$\alpha$ : Tot .81- .88;

Empatía .80- .84;

Autoconcepto .74-

.76; Motivación

.76- .83 ;

Autocontrol .73-

.75 ;

Conoc. de sí .66-
Convergente: Tot. R-: Agresividad reactiva e instrumental.

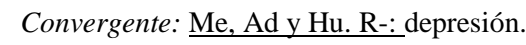

Convergente: Tot.R+: autoexpresión frente a dificultades, decir que no, interacción sexo opuesto, habilidades sociales. R-: trastornos alimenticios, abuso de sustancias, predisposición delincuencia, propensión impulsividad, afecto depresivo, tendencia suicida. Ia.R+: decir que no, habilidades sociales. $\mathrm{R}$-: creencias sexistas. $\underline{\mathrm{Me} . \mathrm{R}+\text { : }}$ autoexpresión frente a dificultades. Ad.R+: decir que no, habilidades sociales, interacción sexo opuesto, defender derechos, autoexpresión frente a dificultades. Hu.R+: defender derechos, autoexpresión frente a dificultades, decir que no, habilidades sociales total. Concurrente: TEIQue-ASF, Regulación MSCEIT.

Convergente: Tot.R-: agresión física, verbal, hostilidad e ira.

Convergente: Tot.R+: extroversión, autoconcepto, rendimiento académico. R-: estrés, manifestaciones emocionales y comportamentales, ansiedad, miedo a evaluaciones, evitación social. Predictiva:Tot.-estrés y ansiedad social. Incremental: supera CI, personalidad y autoconcepto en predecir rendimiento. Concurrente: $\mathrm{EQi}-$ YV y Regulación MSCEIT.

Convergente: Tot.R+: autoeficacia y habilidades sociales.

Convergente: Tot.R+: adaptación académica. $\mathrm{R}-$ : conflicto y hostilidad. Est.R+: nominaciones de amistad.

Convergente: Tot. $\mathrm{R}+\mathrm{t}$ rendimiento. Autoconcepto.R+: ansiedad. Hab. sociales.R-: ansiedad, preocupaciones y dificultades. 
Tabla 2. Continuación

\begin{tabular}{|c|c|c|c|c|}
\hline & & & $\begin{array}{c}.67 ; \\
\text { Hab. sociales .74- } \\
.75 . \\
\text { Spearman-Brown: } \\
\text { Tot: .77- } .84\end{array}$ & \\
\hline & $\begin{array}{l}\text { Referencia a } \\
\text { instrumento original } \\
\text { Dimensión } \\
\text { Regulación }\end{array}$ & $\begin{array}{l}\text { Uno: } 10 \\
\text { Uno: } 5\end{array}$ & $\alpha:$ Tot .71 & $\begin{array}{c}\text { Convergente: Aptitud intelectual. Concurrente: EQi-YV, } \\
\text { TEIQue-ASF. }\end{array}$ \\
\hline $\begin{array}{l}\text { Schutte Self } \\
\text { Report } \\
\text { Inventory } \\
\text { (SSRI; Schutte } \\
\text { et al., 1998) }\end{array}$ & $\begin{array}{l}\text { Española (Chico, } \\
\text { 1999) }\end{array}$ & $\begin{array}{l}\text { Dos: } 40 \text {, } \\
\quad 49\end{array}$ & $\alpha:$ Tot .80 & - \\
\hline $\begin{array}{l}\text { Brief Emotional } \\
\text { Intelligence } \\
\text { Scale-10 } \\
\text { (BEIS-10; } \\
\text { Davies, Lane, } \\
\text { Devonport, \& } \\
\text { Scott, 2010) }\end{array}$ & $\begin{array}{l}\text { (Martín, Guzmán, \& } \\
\text { de Benito, 2018) }\end{array}$ & Uno: 37 & $\begin{array}{c}\alpha: \text { Tot } .75-.80 \\
\text { Test-retest } r: .76 .\end{array}$ & $\begin{array}{c}\text { Convergente: } \text { Tot.R+: clima motivacional y relaciones } \\
\text { positivas con el entrenador. Factorial: AFE IFI .92 TLI } .9 \\
\text { CFI } .92 \text { NFI } .88 \text { RMSEA } .07\end{array}$ \\
\hline $\begin{array}{l}\text { Emotional } \\
\text { Awareness } \\
\text { Questionnaire } \\
\text { (Rieffe, } \\
\text { Oosterveld, } \\
\text { Miers, Terwogt, } \\
\text { \& Ly, 2008) }\end{array}$ & $\begin{array}{l}\text { Cuestionario de } \\
\text { Conciencia } \\
\text { Emocional } \\
\text { (Samper-García, } \\
\text { Mesurado, Richaud, } \\
\text { \& Llorca, 2016) }\end{array}$ & Uno: 59 & $\begin{array}{c}\alpha: \text { Tot .74; } \\
\text { Distinción .70; } \\
\text { Intercambio verbal } \\
.70 ; \text { No } \\
\text { ocultamiento .74; } \\
\text { Conciencia } \\
\text { corporal .72; } \\
\text { Atención a otros } \\
.71 ; \text { Análisis } \\
\text { emocional .68. }\end{array}$ & $\begin{array}{c}\text { Factorial: AFC } 6 \text { factores GFI .94 AGFI .92 CFI .88 RMR } \\
.02 \text { RMSEA } .04\end{array}$ \\
\hline $\begin{array}{l}\text { Facial Action } \\
\text { Coding System } \\
\text { (Ekman \& } \\
\text { Friesen, 2003) }\end{array}$ & $\begin{array}{c}\text { Prueba de } \\
\text { reconocimiento } \\
\text { emocional } \\
\text { (Ambrona, López- } \\
\text { Pérez, \& Márquez- } \\
\text { González, 2012) }\end{array}$ & Uno: 1 & - & - \\
\hline $\begin{array}{l}\text { Prueba de } \\
\text { comprensión } \\
\text { emocional } \\
\text { (García- } \\
\text { Nogales \& } \\
\text { Sotillo, 2006) }\end{array}$ & $\begin{array}{c}\text { Prueba de } \\
\text { comprensión } \\
\text { emocional simple y } \\
\text { mixta (Ambrona, } \\
\text { López-Pérez, \& } \\
\text { Márquez-González, } \\
\text { 2012) }\end{array}$ & Uno: 1 & - & - \\
\hline $\begin{array}{l}\text { PERVALEX } \\
\text { (Mestre, Guil, } \\
\text { Martínez- } \\
\text { Cabañas, } \\
\text { Larrán, \& } \\
\text { González, } \\
\text { 2011) }\end{array}$ & $\begin{array}{c}\text { PERCERVAL } \\
\text { (Ramírez-Lucas, } \\
\text { Ferrando, \& Sáinz, } \\
\text { 2015) }\end{array}$ & Uno: 48 & $\begin{array}{l}\alpha: \text { Ítems fáciles } .80 \\
\text { Ítems complejos } \\
.82\end{array}$ & $\begin{array}{l}\text { Discriminante: } \\
\text { No correlaciona con EQi-360. }\end{array}$ \\
\hline $\begin{array}{l}\text { Self-Rated } \\
\text { Emotional } \\
\text { Intelligence } \\
\text { Scale (SREIS; } \\
\text { Brackett, } \\
\text { Rivers, } \\
\text { Shiffman, } \\
\text { Lerner, \& } \\
\text { Salovey, 2006) }\end{array}$ & $\begin{array}{l}\text { Referencia a } \\
\text { instrumento original }\end{array}$ & Uno: 49 & - & - \\
\hline $\begin{array}{l}\text { Wong and } \\
\text { Law's } \\
\text { Emotional } \\
\text { Intelligence } \\
\text { Scale (WLEIS; } \\
\text { Wong \& Law, } \\
\text { 2002) }\end{array}$ & $\begin{array}{l}\text { Referencia a } \\
\text { instrumento original }\end{array}$ & Uno: 12 & $\begin{array}{l}\alpha: \text { Autoevaluación } \\
\quad(\text { Ae) } .72 ; \\
\text { Evaluación otros } \\
\text { (Eo) } .73 ; \text { Uso } \\
\text { emociones (Ue) } \\
.70 ; \text { Regulación } \\
(\mathrm{Rg}) .78 . \Omega: \text { Ae } \\
.72 ; \text { Eo .72; Ue } \\
.70 ; \operatorname{Rg} .79 . V E:\end{array}$ & $\begin{array}{l}\text { Convergente: } \text { Ae.R-: estrés, manifestaciones emocionales, } \\
\text { miedo a evaluaciones negativas. } \\
\text { Eo.R-: estrés, manifestaciones emocionales, miedo a } \\
\text { evaluaciones negativas, ansiedad. Ue.R-: estrés, } \\
\text { manifestaciones emocionales, miedo a evaluaciones } \\
\text { negativas, evitación social. Rg.R-: estrés, manifestaciones } \\
\text { emocionales y comportamentales, miedo a evaluaciones } \\
\text { negativas, evitación social nuevas situaciones, ansiedad. } \\
\text { Predictiva: Tot- estrés y ansiedad social. }\end{array}$ \\
\hline
\end{tabular}

Ae .5 ; Eo .5; Ue .5; $\operatorname{Rg} .5$ 
Tabla 2. Continuación

Cuestionario E-
motions (Zych,
Ortega-Ruiz, \&
Martín-López,
2017)

Original

Test de

Inteligencia

Emocional de la

Fundación

Botín para

adolescentes

(TIEFBA;

Fernández-

Berrocal, Ruiz-

Aranda,

Salguero,

Palomera, \&

Extremera,

2018)

Test de

Inteligencia

Socio-

Emocional

(Chiriboga, \&

Franco, 2001)

$\alpha:$ Tot .92;
Expresión (Ex)
$.85 ;$ Percepción

5; Percepción

(Pe) 76

Facilitación $(\mathrm{Fa})$

.92; Comprensión

$$
\text { y manejo }
$$

emocional (Cme)

.88. Test-retest $r$ :

Tot .62; Ex .66; Pe

.55 ; Fa .56; Cme

.54 .

$\alpha$ : Tot .98;

Comunicación .90;

Cond. Social .94;

Autoconocimiento

.90; Emociones

.94; Violencia .96;

Solución conflictos

.94; Prosocialidad .94

$\alpha$ : Tot .91;

Percepción .86;

Facilitación .76;

Comprensión .76;

Manejo .74
Convergente: Tot, Ex, Fa.R+: dificultad para identificar emociones. Factorial: AFE 4 factores CFI .98 NFI .95 NNFI .98 RMSEA .05. Concurrente: TMMS-24 (Tot y At con todas las escalas, $\mathrm{Cl}$ con $\mathrm{Cme}$ ).
Convergente: Tot.R-: agresión física y verbal. Incremental: Alta IE predice baja agresión física en seguimiento más que niveles iniciales de agresión física.

Nota a En Hab. social .71

Nota. a. En los datos sobre fiabilidad figuran los rangos entre el coeficiente más bajo reportado y el más alto. Hay estudios en los que no se reporta ningún estadístico y no todos los estadísticos aparecen en todas las investigaciones. Para la información detallada, consultar la fuente original. b. Las correlaciones entre las variables mencionadas son significativas al menos al nivel de $p<.05$. R+: relaciones positivas; R-: relaciones negativas. Para facilitar la lectura de la tabla, en ocasiones se utilizan abreviaciones (ej: nombres de las dimensiones de los instrumentos); en estos casos, se utilizó primero el nombre completo seguido de un paréntesis con la abreviación que fue empleada en el resto de la tabla (ej: Atención (At).).

\section{Discusión}

El objetivo de este estudio fue analizar los instrumentos que están siendo utilizados para evaluar la IE en población infanto-juvenil en el contexto hispanohablante. Los resultados indican que se han utilizado múltiples sistemas de evaluación de la IE en esta población, cuestión que permite afirmar que el panorama es similar al de la evaluación en la población adulta (ver Rodrigo-Ruiz, Cejudo, \& PérezGonzález, 2019). De hecho, a nivel teórico, se observó que las investigaciones se basaban en los distintos modelos que describen el constructo (ej: Bar-On, 2006; Mayer \& Salovey,
1997; Petrides \& Furnham, 2001). Estudios posteriores, en particular de carácter metaanalítico, deberán identificar el potencial papel predictivo de la IE en la infancia y adolescencia desde cada modelo teórico y así unificar criterios conceptuales, analizar el tamaño de efecto del impacto de este constructo en variables de funcionamiento vital y aportar, en la medida de lo posible, claridad a la disparidad de resultados encontrados entre diferentes medidas y enfoques utilizados en etapas infanto-juveniles. Se observó que el período menos estudiado fue la etapa preescolar (3 a 6 años), seguida de la escolar (7 a 11 años) y luego la adolescencia temprana y media (12 a 17 años). En la mayoría de los casos las medidas eran versiones de instrumentos 
Tabla 3. Instrumentos utilizados en cada momento evolutivo

\begin{tabular}{|c|c|c|c|}
\hline $\begin{array}{l}\text { Momento } \\
\text { evolutivo }\end{array}$ & \multicolumn{3}{|c|}{ Instrumentos IE } \\
\hline $\begin{array}{l}\text { Edad } \\
\text { preescolar } \\
\text { (3 a } 6 \text { años) }\end{array}$ & $\begin{array}{l}\text { - Adaptación MSCEIT (Pulido, 2017) } \\
\text { - EQi-360 (Bar-On \& Parker, 2000) } \\
\text { - EQi-YV completo (Ferrándiz, } \\
\text { Hernández, Bermejo, Ferrando, \& Sáinz, } \\
\text { 2012) }\end{array}$ & $\begin{array}{l}\text { - PERCERVAL (Ramírez-Lucas, Ferrando, } \\
\text { \& Sáinz, 2015) } \\
\text { - Prueba de reconocimiento emocional, } \\
\text { comprensión simple y mixta (Ambrona, } \\
\text { López-Pérez, \& Márquez-González, 2012) }\end{array}$ & \\
\hline $\begin{array}{l}\text { Edad escolar } \\
\text { (7 a } 11 \text { años) }\end{array}$ & $\begin{array}{l}\text { - Adaptación MSCEIT (Pulido, 2017) } \\
\text { - E-motions (Zych, Ortega-Ruiz, \& } \\
\text { Marín-López, 2017) } \\
\text { - Test de Inteligencia Socio Emocional } \\
\text { (Chiriboga \& Franco, 2001) }\end{array}$ & $\begin{array}{l}\text { - EQi-YV completo y abreviado (Ferrándiz, } \\
\text { Hernández, Bermejo, Ferrando, \& Sáinz, } \\
\text { 2012; Sáinz, Ferrándiz, Fernández-Vidal, \& } \\
\text { Ferrando, 2014; Ugarriza \& Pajares, 2005) }\end{array}$ & $\begin{array}{l}\text { - TEIQue-ASF (Petrides } \\
\text { et al, 2006) } \\
\text { - TMMS- } 24 \\
\text { (Fernández-Berrocal, } \\
\text { Extremera, \& Ramos, } \\
\text { 2004) } \\
\text { - TMMS (Fernández- } \\
\text { Berrocal et al., 1998) } \\
\text { - TMMS-C (Rockhill \& } \\
\text { Greener, 1999) }\end{array}$ \\
\hline $\begin{array}{l}\text { Adolescencia } \\
\text { temprana } \\
\text { (12 a } 14 \text { años) }\end{array}$ & $\begin{array}{l}\text { - BEIS-10 (Martín, Guzmán \& de Benito, } \\
\text { 2018) } \\
\text { - Cuestionario de Conciencia Emocional } \\
\text { (Samper-García, Mesurado, Richaud, \& } \\
\text { Llorca, 2016) } \\
\text { - EIEI (González-Cabrera, Pérez-Sancho, } \\
\text { \& Calvete, 2016) } \\
\text { - E-motions (Zych, Ortega-Ruiz, \& } \\
\text { Marín-López, 2017) } \\
\text { - TEIQue-ASF (Petrides et al, 2006; } \\
\text { Ferrando et al., 2011) }\end{array}$ & $\begin{array}{l}\text { - EQi-YV completo y abreviado (Esnaola, } \\
\text { Freeman, Sarasa, Fernández-Zabala, \& } \\
\text { Axpe, 2016; Ferrándiz, Hernández, } \\
\text { Bermejo, Ferrando, \& Sáinz, 2012; Sáinz, } \\
\text { Ferrándiz, Fernández-Vidal, \& Ferrando, } \\
\text { 2014; Rucalva, Gallegos, Lorenzo, \& } \\
\text { Borges, 2014; Ugarriza \& Pajares, 2005) } \\
\text { - MSCEIT adaptaciones (Extremera \& } \\
\text { Fernández-Berrocal, 2002; Pulido, 2017) } \\
\text { - SSRI (Schutte et al., 1998) } \\
\text { - TEIQue-SF (Petrides, 2012) }\end{array}$ & $\begin{array}{l}\text { - TIEFBA (Fernández- } \\
\text { Berrocal, Ruiz-Aranda, } \\
\text { Salguero, Palomera, \& } \\
\text { Extremera, 2018) } \\
\text { - TMMS- 24 } \\
\text { (Fernández-Berrocal, } \\
\text { Extremera, \& Ramos, } \\
\text { 2004) } \\
\text { - TMMS-23 (Salguero, } \\
\text { Fernández-Berrocal, } \\
\text { Balluerka, \& Aritzeta, } \\
\text { 2010) } \\
\text { - TMMS (Fernández- } \\
\text { Berrocal et al., 1998) } \\
\text { - TMMS- 21 (Calero, } \\
\text { 2013) } \\
\text { - TMMS- 12 (Gómez- } \\
\text { Baya, Mendoza, \& } \\
\text { Paino, 2016) } \\
\text { - WLEIS (Wong \& } \\
\text { Law, 2002) }\end{array}$ \\
\hline $\begin{array}{l}\text { Adolescencia } \\
\text { media } \\
\text { (15 a } 17 \text { años) }\end{array}$ & $\begin{array}{l}\text { - BEIS-10 (Martín, Guzmán, \& de Benito, } \\
\text { 2018) } \\
\text { - Cuestionario de Conciencia Emocional } \\
\text { (Samper-García, Mesurado, Richaud, \& } \\
\text { Llorca, 2016) } \\
\text { - Cuestionario de Evaluación del } \\
\text { Programa (Garaigordobil, 2010) } \\
\text { - EIEI (González-Cabrera, Pérez-Sancho, } \\
\text { \& Calvete, 2016) } \\
\text { - E-motions (Zych, Ortega-Ruiz, \& } \\
\text { Marín-López, 2017) } \\
\text { - TEIQue-ASF (Petrides et al, 2006; } \\
\text { Ferrando et al., 2011) }\end{array}$ & $\begin{array}{l}\text { - EQi-YV completo y abreviado (Esnaola, } \\
\text { Freeman, Sarasa, Fernández-Zabala, \& } \\
\text { Axpe, 2016; Ferrándiz, Hernández, } \\
\text { Bermejo, Ferrando, \& Sáinz, 2012; Sáinz, } \\
\text { Ferrándiz, Fernández-Vidal, \& Ferrando, } \\
\text { 2014; Rucalva, Gallegos, Lorenzo, \& } \\
\text { Borges, 2014; Ugarriza \& Pajares, 2005) } \\
\text { - MSCEIT original y adaptación (Pulido, } \\
\text { 2017) } \\
\text { - SREIS (Brackett, Rivers, Shiffman, } \\
\text { Lerner, \& Salovey, 2006) } \\
\text { - SSRI (Schutte et al., 1998) }\end{array}$ & $\begin{array}{l}\text { - TEIQue-SF (Petrides, } \\
\text { 2012) } \\
\text { - TIEFBA (Fernández- } \\
\text { Berrocal, Ruiz-Aranda, } \\
\text { Salguero, Palomera, \& } \\
\text { Extremera, 2018) } \\
\text { - TMMS- 24 } \\
\text { (Fernández-Berrocal, } \\
\text { Extremera, \& Ramos, } \\
\text { 2004) } \\
\text { - TMMS-23 (Salguero, } \\
\text { Fernández-Berrocal, } \\
\text { Balluerka, \& Aritzeta, } \\
\text { 2010) } \\
\text { - TMMS (Fernández- } \\
\text { Berrocal et al., 1998) } \\
\text { - TMMS- 21 (Calero, } \\
\text { 2013) } \\
\text { - TMMS- 12 (Gómez- } \\
\text { Baya, Mendoza, \& } \\
\text { Paino, 2016) } \\
\text { - WLEIS (Wong \& } \\
\text { Law, 2002) }\end{array}$ \\
\hline
\end{tabular}


Tabla 4. Instrumentos para la evaluación de la inteligencia emocional en castellano con adaptaciones publicadas para población infanto-juvenil

\begin{tabular}{|c|c|c|c|c|c|c|}
\hline $\begin{array}{l}\text { Modelo } \\
\text { teórico IE }\end{array}$ & $\begin{array}{l}\text { Instrumento } \\
\text { original }\end{array}$ & $\begin{array}{c}\text { Primer adaptación al } \\
\text { castellano en población } \\
\text { infanto-juvenil }\end{array}$ & $\begin{array}{l}\text { País de la } \\
\text { adaptación }\end{array}$ & $\begin{array}{l}\text { Número de } \\
\text { ítems }\end{array}$ & Dimensiones & $\begin{array}{l}\text { Muestra de la } \\
\text { adaptación }\end{array}$ \\
\hline $\begin{array}{l}\text { Habilidades (Mayer } \\
\text { \& Salovey, 1997) }\end{array}$ & $\begin{array}{c}\text { Trait Meta Mood } \\
\text { Scale (TMMS; } \\
\text { Salovey, Mayer, } \\
\text { Goldman, Turvey, \& } \\
\text { Palfai, 2002) }\end{array}$ & $\begin{array}{c}\text { TMMS- } 23 \text { (Salguero, } \\
\text { Fernández-Berrocal, } \\
\text { Balluerka, \& Aritzeta, } \\
\text { 2010) }\end{array}$ & España & 23 ítems & $\begin{array}{l}3 \text { (atención, claridad y } \\
\text { reparación emocional) }\end{array}$ & $\begin{array}{c}1497 \text { adolescentes, } \\
\text { de } 12 \text { a } 17 \text { años }\end{array}$ \\
\hline $\begin{array}{l}\text { Habilidades (Mayer } \\
\text { \& Salovey, 1997) }\end{array}$ & $\begin{array}{l}\text { Mayer-Salovey- } \\
\text { Caruso Emotional } \\
\text { Intelligence Test } \\
\text { (MSCEIT; Mayer, } \\
\text { Salovey, \& Caruso, } \\
\text { 2002) }\end{array}$ & $\begin{array}{c}\text { Sin nombre (Pulido, } \\
\text { 2017) }\end{array}$ & España & 58 ítems & $\begin{array}{l}5 \text { (conocimiento de sí } \\
\text { mismos, motivación, } \\
\text { autoconcepto, empatía, } \\
\text { autocontrol) }\end{array}$ & $\begin{array}{c}404 \text { NNA, de } 6 \text { a } 12 \\
\text { años }\end{array}$ \\
\hline $\begin{array}{l}\text { Habilidades (Mayer } \\
\text { \& Salovey, 1997) }\end{array}$ & $\begin{array}{l}\text { Schutte Self Report } \\
\text { Inventory (SSRI; } \\
\text { Schutte et al., 1998) }\end{array}$ & $\begin{array}{c}\text { SSRI } \\
\text { (Omar, Salessi, Urteaga, } \\
\text { \& Vaamonde, 2014) }\end{array}$ & Argentina & 25 ítems & $\begin{array}{l}2 \text { (expresión y regulación y } \\
\text { usos de la emoción) }\end{array}$ & $\begin{array}{l}1890 \text { adolescentes y } \\
\text { jóvenes de } 15 \text { a } 26 \\
\text { años }\end{array}$ \\
\hline $\begin{array}{l}\text { Habilidades (Mayer } \\
\text { \& Salovey, 1997) }\end{array}$ & $\begin{array}{l}\text { Brief Emotional } \\
\text { Intelligence Scale-10 } \\
\text { (BEIS-10; Davies, } \\
\text { Lane, Devonport, \& } \\
\text { Scott, 2010) }\end{array}$ & $\begin{array}{c}\text { BEIS-10 } \\
\text { (Martín, Guzmán, \& de } \\
\text { Benito, 2018) }\end{array}$ & España & 10 ítems & $\begin{array}{c}5 \text { (evaluación de las } \\
\text { propias emociones, } \\
\text { evaluación de las emociones } \\
\text { de los demás, } \\
\text { regulación de las propias } \\
\text { emociones, regulación de las } \\
\text { emociones } \\
\text { de los demás y utilización de } \\
\text { las emociones) }\end{array}$ & $\begin{array}{l}327 \text { adolescentes de } \\
12 \text { a } 16 \text { años }\end{array}$ \\
\hline $\begin{array}{l}\text { Habilidades (Mayer } \\
\text { \& Salovey, 1997) }\end{array}$ & $\begin{array}{l}\text { PERVALEX (Mestre, } \\
\text { Guil, Martínez- } \\
\text { Cabañas, Larrán, \& } \\
\text { González, 2011) * }\end{array}$ & $\begin{array}{l}\text { PERCERVAL (Ramírez- } \\
\text { Lucas, Ferrando, \& Sáinz, } \\
\text { 2015) }\end{array}$ & España & 16 ítems & $\begin{array}{l}2 \text { (ítems sencillos e ítems } \\
\text { complejos) }\end{array}$ & $\begin{array}{c}83 \text { niños y niñas de } \\
5 \text { años }\end{array}$ \\
\hline $\begin{array}{l}\text { Habilidades (Mayer } \\
\text { \& Salovey, 1997) }\end{array}$ & $\begin{array}{l}\text { Wong and Law's } \\
\text { Emotional } \\
\text { Intelligence Scale } \\
\text { (WLEIS; Wong \& } \\
\text { Law, 2002) }\end{array}$ & $\begin{array}{c}\text { WLEIS } \\
\text { (Cejudo, Rodrigo-Ruiz, } \\
\text { López-Delgado,\& } \\
\text { Losada, 2018) }\end{array}$ & España & 16 ítems & $\begin{array}{l}4 \text { (valoración y expresión de } \\
\text { las emociones propias, } \\
\text { valoración y reconocimiento } \\
\text { de las emociones en otros, } \\
\text { regulación de las propias } \\
\text { emociones y uso de la } \\
\text { emoción para facilitar el } \\
\text { desempeño) }\end{array}$ & $\begin{array}{l}505 \text { adolescentes de } \\
12 \text { a } 18 \text { años }\end{array}$ \\
\hline $\begin{array}{l}\text { Habilidades (Mayer } \\
\text { \& Salovey, 1997) }\end{array}$ & $\begin{array}{c}\text { Cuestionario E- } \\
\text { motions (Zych, } \\
\text { Ortega-Ruiz, \& } \\
\text { Martín-López, 2017) }\end{array}$ & Original en castellano & España & 21 ítems & $\begin{array}{l}4 \text { (expresión, percepción, } \\
\text { facilitación y uso y manejo } \\
\text { emocional) }\end{array}$ & $\begin{array}{l}2139 \text { adolescentes } \\
\text { de } 11 \text { a } 19 \text { años }\end{array}$ \\
\hline $\begin{array}{l}\text { Habilidades (Mayer } \\
\text { \& Salovey, 1997) }\end{array}$ & $\begin{array}{c}\text { Test de Inteligencia } \\
\text { Emocional de la } \\
\text { Fundación Botín para } \\
\text { adolescentes }\end{array}$ & Original en castellano & España & 8 escenas & $\begin{array}{c}4 \text { (percepción, facilitación, } \\
\text { comprensión y regulación } \\
\text { emocional) }\end{array}$ & $\begin{array}{l}1684 \text { adolescentes } \\
\text { de } 12 \text { a } 17 \text { años }\end{array}$ \\
\hline
\end{tabular}

(TIEFBA; FernándezBerrocal, RuizAranda, Salguero, Palomera, \& Extremera, 2018)

Mixto (Bar-On, Emotional Quotient 2006) inventory- Youth version (EQi-YV; Bar-On \& Parker, 2000)

Rasgo (Petrides y Furnham, 2001)

\section{Conciencia} emocional (Rieffe, Oosterveld, Miers, Terwogt, \& Ly, 2008)

\section{Trait Emotional Intelligence} Questionnaire-

Adolescent Short

Form (TEIQue-ASF; Petrides et al., 2006)

Emotional Awareness Questionnaire (Rieffe, Oosterveld, Miers, Terwogt, \& Ly, 2008)
Versiones completa y abreviada (Ugarriza \& Pajares, 2005)

TEIQue-ASF (Ferrando et al., 2011)

Cuestionario de Conciencia Emocional (Samper-García, Mesurado, Richaud, \& Llorca, 2016)
Completa: 60 ítems; ánimo general); Abreviada: 4 (intrapersonal, interpersonal, adaptabilidad, manejo del estrés)

España $\quad 30$ ítems 1 (inteligencia emocional 290 adolescentes, de rasgo)

España $\quad 30$ ítems

6 (distinción de las emociones, intercambio verbal de las emociones, no

ocultamiento de las emociones, conciencia corporal, análisis de las emociones y atención a las emociones de los demás)

5 (autoconciencia, autocontrol, 10 a 12 años de 14 a 16 años

18 años

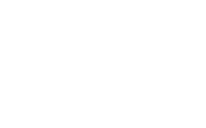
10 años
1316 adolescentes 
Tabla 4. Continuación

\begin{tabular}{|c|c|c|c|c|c|c|}
\hline \multirow[b]{2}{*}{ No se indica } & \multicolumn{3}{|l|}{$\begin{array}{c}\text { (Chiriboga \& Franco, } \\
\text { 2001) }\end{array}$} & \multicolumn{3}{|c|}{$\begin{array}{l}\text { aprovechamiento emocional, } \\
\text { empatía y habilidad social) }\end{array}$} \\
\hline & $\begin{array}{l}\text { Facial Action Coding } \\
\text { System (Ekman \& } \\
\text { Friesen, 2003) }\end{array}$ & $\begin{array}{l}\text { Prueba de reconocimiento } \\
\text { emocional (Ambrona, } \\
\text { López-Pérez, \& Márquez- } \\
\text { González, 2012) }\end{array}$ & España & 14 fotografías & NA & $\begin{array}{c}60 \text { niños y niñas de } \\
6 \text { a } 7 \text { años }\end{array}$ \\
\hline No se indica & $\begin{array}{c}\text { Prueba de } \\
\text { comprensión } \\
\text { emocional (García- } \\
\text { Nogales \& Sotillo, } \\
\text { 2006) * }\end{array}$ & $\begin{array}{l}\text { Prueba de comprensión } \\
\text { emocional simple y mixta } \\
\text { (Ambrona, López-Pérez, } \\
\text { \& Márquez-González, } \\
\text { 2012) }\end{array}$ & España & $\begin{array}{l}\text { Simple: } 15 \\
\text { historias. } \\
\text { Mixta: } 6 \\
\text { historias }\end{array}$ & NA & $\begin{array}{c}60 \text { niños y niñas de } \\
6 \text { a } 7 \text { años }\end{array}$ \\
\hline No se indica & $\begin{array}{c}\text { Cuestionario de } \\
\text { evaluación del } \\
\text { programa } \\
\text { (Garaigordobil, 2010) }\end{array}$ & Original en castellano & España & 48 ítems & $\begin{array}{c}8 \text { (comunicación intragrupo, } \\
\text { conducta social, } \\
\text { autoconcepto, imagen de los } \\
\text { demás y de los seres } \\
\text { humanos, emociones, } \\
\text { violencia, solución de } \\
\text { conflictos, valores } \\
\text { prosociales y derechos } \\
\text { humanos y bienestar } \\
\text { psicológico subjetivo) }\end{array}$ & $\begin{array}{l}216 \text { adolescentes de } \\
15 \text { a } 17 \text { años }\end{array}$ \\
\hline
\end{tabular}

Nota NNA= niños, niñas y adolescentes.NA = No aplica porque el instrumento no tiene dimensiones o factores. $*$ Se señala con asterisco cuando el instrumento original también fue diseñado en castellano.

originalmente diseñados para población adulta, por lo cual se emplearon formas habituales para su evaluación, como cuestionarios y pruebas de habilidad (Cobos-Sánchez, Flujas-Contreras, \& Gómez-Becerra, 2017; Ferrando et al., 2011).

De hecho, al analizar los instrumentos en sí, se observó que los más utilizados fueron adaptaciones de instrumentos para adultos, como el TMMS-24 en primer lugar (FernándezBerrocal, Extremera, \& Ramos, 2004) y el EQiYV, en segundo lugar (Ferrándiz, Hernández, Bermejo, Ferrando, \& Sáinz, 2012). Estos cuestionarios mostraron mayor respaldo a nivel psicométrico ya que más investigaciones reportan datos al respecto. Sobre el TMMS-24, se trata de una prueba fiable, que refleja la estructura factorial original del instrumento Salovey, Mayer, Goldman, Turvey, \& Palfai, 1995) y numerosos estudios reflejan su validez de criterio y de constructo (ver Tabla 2). A pesar de ello, habiendo una versión adaptada para adolescentes (TMMS-23; Salguero, Fernández-Berrocal, Balluerka, \& Aritzeta, 2010) se sigue eligiendo utilizar la versión de adultos. Esta práctica no permite fortalecer la calidad de la evaluación en adolescentes. Por otra parte, el EQi-YV también posee cualidades satisfactorias en cuanto a fiabilidad y validez. No obstante, la fiabilidad tiende a disminuir cuando se utiliza con población infantil, aspecto que será discutido más adelante.

En líneas generales, se observó que casi todos los instrumentos utilizados mostraban altos niveles de consistencia interna y que, en los casos en los que se reportaba, los datos sobre validez también eran prometedores (Tabla 2). Sin embargo, se considera que existen mediciones que tienen menor evidencia psicométrica con la población diana. Por ejemplo, la adaptación de varios instrumentos fue conducida con muestras relativamente pequeñas para constituir un proceso adecuado de adaptación (ej: Test de Inteligencia Socioemocional de Chiriboga y Franco (1999) o la Prueba de reconocimiento y comprensión emocional simple y mixta de Ambrona, LópezPérez, \& Márquez-González, 2012). En otras medidas el análisis factorial que realizan los autores no refleja el modelo teórico en el que se respaldan (ej: la adaptación del MSCEIT de Pulido, 2017 o el PERCERVAL de RamírezLucas, Ferrando, \& Sáinz, 2015). Algunos instrumentos no están basados en un modelo de inteligencia emocional específico (ej: cuestionario de evaluación del programa de Garaigordobil, 2010). Por otra parte, hay sistemas de evaluación que cumplen con estos criterios (i.e. muestras amplias, basados en un modelo de IE concreto y cuyos resultados reflejan la estructura factorial del mismo) y que han sido adaptados o diseñados específicamente para adolescentes (ej: E-motions de Zych, Ortega-Ruiz y Martín-López (2017) o el TIEFBA de Fernández-Berrocal, Ruiz-Aranda, Salguero, Palomera, \& Extremera, 2018). Lejos de pretender dar un juicio de valor sobre los instrumentos hallados, la intención es reflejar la necesidad de continuar la investigación con todas estas medidas para robustecer su calidad.

En otro orden de ideas, al tener presente la 
acelerada curva de desarrollo y la gran variabilidad existente desde la infancia a la adolescencia, se considera que no se puede abordar períodos tan dispares de la misma manera. Un buen sistema de evaluación de la IE debe atender no sólo a las propiedades psicométricas, sino también al nivel de desarrollo característico de la población a la que se va a evaluar. De hecho, tal como se mencionó anteriormente, en los artículos revisados se encontró que cuando se utilizaron versiones que no estaban validadas para infantes o adolescentes, éstas mostraban baja fiabilidad o resultados inconsistentes (ej: Ferrándiz, Hernández, Bermejo, Ferrando y Sáinz, 2012; Jiménez \& López-Zafra, 2011; Mestre, Guil, Lopes, Salovey, \& Gil-Olarte, 2006; Ugarriza \& Pajares, 2005). Esto lleva a valorar si en estas edades se tiene el desarrollo social y cognitivo necesario para conocer y reportar los propios procesos emocionales (Ferrando et al., 2011; Jiménez \& López-Zafra, 2011).

Una vía para solventar estas flaquezas podría ser el ceñirse a un modelo teórico que permita evaluar la IE a lo largo de la vida y, a partir de él, generar ítems diferentes que reflejen la misma habilidad latente, tal como sugieren Denham, Wyatt, Bassett, Echeverria y Knox (2009). Por ejemplo, desde el modelo de Mayer y Salovey (1997) se plantea que cada rama de la IE se construye de manera progresiva y que existen diversas áreas de razonamiento que se van desarrollando a lo largo de la vida (Mayer, Caruso, \& Salovey, 2016). Se entiende, entonces, que no todas las capacidades son igual de importantes o se expresan de la misma forma en todos los momentos evolutivos (Denham \& Kochanoff, 2002; Halle \& Darling-Churchill, 2016).

Otra línea para investigaciones futuras que pretendan mejorar la calidad de los instrumentos para medir la IE implica determinar cuáles son las mejores formas de evaluación atendiendo al nivel de desarrollo cognitivo, por ejemplo en cuanto a la capacidad de atención, de lectura, de descentramiento y de introspección (Denham \& Kochanoff, 2002; Halle \& Darling-Churchill, 2016). Algunas de estas características fueron tomadas en cuenta en las pruebas de habilidades en la infancia (ej: Ambrona, López-Pérez, \& Márquez-González, 2012; Ramírez-Lucas,
Ferrando, \& Sáinz, 2015). De hecho, en las Tablas 3 y 4 se puede observar que muy pocos instrumentos han sido validados para población infantil, por lo cual sigue siendo un área poco investigada.

Dada la importancia de los pares para el desarrollo emocional (Denham \& Kochanoff, 2002), estudios futuros podrían incluir la perspectiva de diversos informantes, ya que cada uno brinda información única del sujeto (Humphrey et al., 2011) y el comportamiento infantil y adolescente tiende a estar influenciado por el contexto (Denham, Wyatt, Bassett, Echeverria, \& Knox, 2009). De los artículos revisados, en sólo uno se incluyó la perspectiva de los padres como informantes externos (RamírezLucas, Ferrando, \& Sáinz, 2015). Asimismo, se podrían incluir los aportes de niños, niñas y/o adolescentes, de padres, madres y de profesionales de la educación en el proceso de diseño y/o validación para mejorar la comprensión de la evaluación y aumentar su validez ecológica (Correia, Marques, \& Roberto, 2017; FernándezBerrocal, Ruiz-Aranda, Salguero, Palomera, \& Extremera, 2018). También cabría preguntarse si las áreas de la IE o temas que se exploran con las mediciones tienen los mismos significados en las distintas poblaciones con las que se trabaja, cuestión que se aplica tanto al nivel de desarrollo evolutivo del sujeto así como al contexto cultural en el que se realiza la evaluación.

El presente estudio ha tenido algunas limitaciones. Dada la controversia conceptual del campo, es posible que utilizando términos similares u otras interpretaciones de los criterios de inclusión, los resultados hubieran sido ligeramente diferentes, incluyendo algún estudio adicional. Por otro lado, el no incluir artículos de otras fuentes puede haber llevado a dejar de lado investigaciones o autores referentes en el área. Igualmente, en esta revisión solamente fue posible que una investigadora extrajese y codificase la información. En futuras investigaciones, se recomienda que este proceso sea realizado por al menos dos evaluadores independientes, para aumentar la fiabilidad de los resultados. Finalmente, es posible que debido a sesgos de publicación no se haya accedido a artículos con abordajes distintos de la evaluación y/o el desarrollo emocional. A pesar de estas 
limitaciones, en general, se considera que se abordaron numerosos estudios, de diversos contextos y realidades, haciendo referencia a una gran cantidad de instrumentos, lo cual permite conocer de forma más comprehensiva el estado del arte sobre la evaluación infanto-juvenil de la IE.

Algunas implicaciones sobre los resultados de la presente revisión refieren a su aplicación en el ámbito de la investigación y la intervención. Así por ejemplo, en el campo de la educación, la medición de la IE puede servir para identificar estudiantes que necesiten apoyo adicional o que tienen niveles atípicos de desarrollo emocional (Wigelsworth, Humphrey, Kalambouka, \& Lendrum, 2010). En el ámbito clínico, existen autores que consideran que los déficits en el procesamiento emocional pueden ser la base de la psicopatología infantil, por lo que la evaluación emocional está vinculada al diagnóstico, la identificación de técnicas adecuadas de tratamiento y la valoración continua sobre la utilidad del tratamiento elegido (Zeman, Klimes, Cassano, \& Adrian, 2007). Una medición rigurosa de los niveles de IE puede constituir un método de cribado para detectar déficits emocionales en niños, niñas o adolescentes en distintos entornos, por lo que resulta necesario contar con instrumentos válidos y fiables.

\section{Referencias}

Los artículos señalados con asterisco están incluidos en la revisión sistemática. El número en paréntesis indica la referencia utilizada en el cuerpo del artículo.

*(1) Ambrona, T., López-Pérez, B., \& Márquez-

González, M. (2012). Eficacia de un programa

de educación emocional breve para incrementar la competencia emocional de niños de educación primaria. Revista Española de Orientación y Psicopedagogía, 23(1), 39-49. doi:10.5944/reop.vol.23.num.1.2012.11392

Arguís, R., Bolsas, A., Hernández-Paniellos, S., \& Salvador, M. (2012). Programa "Aulas felices”. Psicología positiva aplicada a la Educación.
*(2) Azpiazu, L., Esnaola, I., \& Sarasa, M. (2015). Capacidad predictiva del apoyo social en la inteligencia emocional de adolescentes. European Journal of Education and Psychology, 8(1), 23-29. doi:10.1016/j.ejeps.2015.10.003

*(3) Barahona, L., \& Alegre, A. (2016). Inteligencia emocional y TDAH: Un análisis comparativo en estudiantes de Lima Metropolitana. Propósitos y Representaciones, 4(1), 61-87. doi:10.20511/pyr2016.v4n1.87

Bar-On, R. (2006). The Bar-On model of emotional-social intelligence (ESI). Psicothema, 18, 3-25.

Bar-On, R., \& Parker, J. (2000). The Bar-On Emotional Quotient Inventory: Youth Version (EQ-i:YV) Technical Manual (Translated to Spanish by Caraballo, C. y Villegas, O.).Toronto, Canada: Multi-Health Systems.

*(4) Barrón-Sánchez, M., \& Molero, D. (2014). Estudio sobre inteligencia emocional y afectos en escolares de educación primaria. Revista Electrónica de Investigación y Docencia, 12, 7-18.

*(5) Belmonte, V., Parodi, A., Bermejo, R., Ruiz, M., \& Sáinz, M. (2017). Relaciones entre aptitud intelectual, inteligencia emocional y creatividad en alumnado de ESO. International Journal of Developmental and Educational Psychology, 1(1), 35-44. doi:10.17060/ijodaep.2017.n1.v1.896

Bisquerra, R. (2009). Psicopedagogía de las emociones. Madrid: Síntesis.

Brackett, M., Rivers, S., Shiffman, S., Lerner, N., \& Salovey, P. (2005). What is the best way to measure emotional intelligence? A case for performance measures. Hauppauge, New York: Nova Science Publishing.

*(6) Bustamante, J., Barona, E., \& del Barco, B. (2010). Relación entre apego e inteligencia emocional en adolescentes. Revista Electrónica de Motivación y Emoción, 13(34), 8.

*(7) Calero, A. (2013). Versión Argentina de la Trait Meta Mood Scale (TMMS) para adolescentes: Una medida de la inteligencia emocional percibida.Panamerican Journal of Neuropshychology, 7(1), 104-119. doi:10.7714/cnps/7.1.206 
*(8) Calero, A., Barreyro, J., \& Injoque-Ricle, I. (2017). Inteligencia emocional durante la adolescencia: $\mathrm{Su}$ relación con la participación en actividades extracurriculares. Psicodebate, 17(2), 43-54. doi:10.18682/pd.v17i2.693

*(9) Carrillo, A., Estévez, C., \& Gómez-Medina, M. (2018). ¿Influyen las prácticas educativas en el desarrollo de la inteligencia emocional de sus hijos? International Journal of Developmental and Educational Psychology, 1(1), 203-212. doi:10.5944/ap.12.1.14314. 65

*(10) Castañeda, O., Ramírez, S., Rivera, B., \& Merchán, V. (2014). La inteligencia emocional de los estudiantes del grado once de una Institución Educativa de Envigado. Psicoespacios, 8(13), 179-201. doi: $10.25057 / 21452776.327$

*(11) Cejudo, J. (2017). Efectos de un programa de mejora de la inteligencia emocional sobre el ajuste psicosocial y el rendimiento académico en educación primaria. Journal for the Study of Education and Development, 40(3), 516-530. doi:10.1080/02103702.2017.1341099

*(12) Cejudo, J., Rodrigo-Ruiz, D., LópezDelgado, M., \& Losada, L. (2018). Emotional intelligence and its relationship with levels of social anxiety and stress in adolescents. International Journal of Environmental Research and Public Health, 15(6), 1073. doi:10.3390/ijerph15061073.

Chico, E. (1999). Evaluación psicométrica de una escala de la inteligencia emocional. Boletín de Psicología, 62, 65-78.

Chiriboga, R., \& Franco, J. (2001). Validación de un test de Inteligencia Emocional en niños de diez años de edad. Revista Médico de Familia, 9, 24-38

*(13) Cobos-Sánchez, L., Flujas-Contreras, J., \& Gómez-Becerra, I. (2016). The role of emotional intelligence in psychological adjustment among adolescents. Anales de Psicología, 33(1), 66-73.

doi:10.6018/analesps.33.1.240181

*(14) Cobos-Sánchez, L., Flujas-Contreras, J., \& Gómez-Becerra, I. (2017). Intervención en flexibilidad psicológica como competencia emocional en adolescentes: Una serie de casos. Revista de Psicología Clínica con Niños y Adolescentes, 4(2), 135-141.
Correia, A., Marques, A., \& Roberto, M. (2017). Adaptação na transição para a escola: Estudo do Questionário de Adaptação Escolar e da Escala de Competência Social do SSBS2. Revista Iberoamericana de Diagnóstico y Evaluación - e Avaliação Psicológica, 3(45), 17-31. doi:10.21865/RIDEP45.3.02

Davies, K., Lane, A., Devonport, T., \& Scott, J. (2010). Validity and reliability of a Brief Emotional Intelligence Scale (BEIS10). Journal of Individual Differences, 31(4), 198208. doi: 10.1027/1614-0001/a000028

Denham, S., Wyatt, T., Bassett, H., Echeverria, D., \& Knox, S. (2009). Assessing socialemotional development in children from a longitudinal perspective. Journal of Epidemiology y Community Health, 63(1), i37-i52. doi:10.1136/jech.2007.070797

Denham, S., \& Kochanoff, A. (2002). "Why is she crying?" Children's understanding of emotion from preschool to preadolescence. En L. Feldman y P. Salovey, The wisdom in feeling. Psychological processes in Emotional Intelligence, pp. 241-270. New York: Guildford Press.

*(15) Díaz-Castela, M., Hale, W., Muela, J., Espinosa, L., Klimstra, T., \& García, L. (2013). The measurement of perceived emotional intelligence for Spanish adolescents with social anxiety disorder symptoms. Anales de Psicología, 29(2), 509-515.

doi:10.6018/analesps.29.2.144271

*(16) Domínguez-Alonso, J., DomínguezRodríguez, V., López-Pérez, E., \& del Mar, M. (2016). Motivación e inteligencia emocional en estudiantes de Educación Secundaria Obligatoria. Revista de Estudios e Investigación en Psicología y Educación, 3(2), 94-101. doi:10.17979/reipe.2016.3.2.1801

Ekman, P., \& Friesen, W. (2003). Unmasking the Face. A Guide to Recognizing Emotions from Facial Clues. Los Altos, CA: Malor Books.

*(17) Elipe, P., Ortega, R., Hunter, S., \& Del Rey, R. (2012). Inteligencia emocional percibida e implicación en diversos tipos de acoso escolar. Psicología Conductual, 20(1), 169181.

Esnaola, I., Freeman, J., Sarasa, M., FernándezZabala, A., \& Axpe, A. (2016). Validity evidence based on internal structure of scores 
of the Emotional Quotient-Inventory: Youth Version Short (EQ-i: YV-S) in a Spanish Sample. The Spanish Journal of Psychology, 19, 1-9. doi:10.1017/sjp.2016.12

*(18) Esnaola, I., Revuelta, L., Ros, I., \& Sarasa, M. (2017). The development of emotional intelligence in adolescence. Anales de Psicología, 33(2), 327-333.

doi:10.6018/analesps.33.2.251831

*(19) Estévez, C., Carrillo, A., \& Gómez-Medina, M. (2018). Inteligencia emocional y bullying en escolares de primaria. International Journal of Developmental and Educational Psychology, 1(1), 227-238.

doi:10.17060/ijodaep.2018.n1.v1.1200

Extremera, N., \& Fernández-Berrocal, P. (2002). Cuestionario MSCEIT (Versión española 2.0) de Mayer, Salovey y Caruso. Toronto, Canada: Multi-Health Systems Publishers.

Extremera, N., \& Fernández-Berrocal, P. (2007). Una guía práctica de los instrumentos actuales de evaluación de la inteligencia emocional. En J. Mestre y P. Fernández-Berrocal(coords.), Manual de Inteligencia Emocional (pp. 97120). Madrid: Pirámide.

*(20) Fariña, F., Arce, R., \& Novo, M. (2008). Neighborhood and community factors: Effects on deviant behavior and social competence. The Spanish Journal of Psychology, 11(1), 7884. doi: 10.1017/s1138741600004133

*(21) Fernández-Angulo, A., Quintanilla, L., \& Giménez-Dasi, M. (2016). Dialogando sobre emociones con niños en riesgo de exclusión social: un estudio preliminar. Acción Psicológica, 13(1), 191-206. doi:10.5944/ap.13.1.15787

Fernández-Berrocal, P., Alcaide, R., Domínguez, E., Fernández-McNally, C., Ramos, N., \& Ravira, M. (1998, April-May). Adaptación al castellano de la escala Rasgo de Metaconocimiento sobre Estados Emocionales de Salovey et al.: datos preliminares. $V$ Congreso de Evaluación Psicológica, Málaga (Spain).

*(22) Fernández-Berrocal, P., \& Extremera, N. (2003). ¿En qué piensan las mujeres para tener un peor ajuste emocional? Encuentros en Psicología Social, 1(5), 255-259.

Fernández-Berrocal, P., \& Extremera, N. (2016). Ability emotional intelligence, depression, and well-being. Emotion Review, 8(4), 311315. doi:10.1177/1754073916650494

Fernández-Berrocal, P., Extremera, N., \& Ramos, N. (2004). Validity and reliability of the Spanish modified version of the Trait MetaMood Scale. Psychological Reports, 94, 751755. doi:0.2466/pr0.94.3.751-755

Fernández-Berrocal, P., Ruiz-Aranda, D., Salguero, J., Palomera, R., \& Extremera, N. (2018). La relación del Test de Inteligencia Emocional de la Fundación Botín (TIEFBA) con el ajuste personal y escolar de adolescentes españoles. Revista de Psicodidáctica, 23(1), 1-8. doi:10.1016/j.psicod.2017.07.001

*(23) Ferrándiz, C., Hernández, D., Bermejo, R., Ferrando, M., \& Sáinz, M. (2012). Social and emotional intelligence in childhood and adolescence: Spanish validation of a measurement instrument. Revista de Psicodidáctica, 17(2). doi:0.1387/Rev.Psicodidact.2814

*(24) Ferrando, M., Prieto, M., Almeida, L., Ferrándiz, C., Bermejo, R., López-Pina, J. A., ... \& Fernández, M. (2011). Trait emotional intelligence and academic performance: Controlling for the effects of IQ, personality, and self-concept. Journal of Psychoeducational Assessment, 29(2), 150159. doi:10.1177/0734282910374707.

*(25) Fulquez, S., Alguacil, M., \& Pañellas, M. (2011). La atención emocional y la relación con la autoestima, la autoconfianza y las relaciones con los padres y con los pares: Un estudio en adolescentes españoles y mexicanos. International Journal of Developmental and Educational Psychology, 1(2), 403-412.

*(26) Garaigordobil, M. (2010). Efectos del programa" Dando pasos hacia la paz" sobre factores cognitivos y conductuales de la violencia juvenil. Psicología Conductual, 18(2), 277-295.

*(27) Garaigordobil, M., \& Peña, A. (2014). Intervención en las habilidades sociales: Efectos en la inteligencia emocional y la conducta social. Psicología Conductual, 22(3), 551-567.

*(28) Garaigordobil, M., Aliri, J., MartínezValderrey, V., Maganto, C., Bernaras, E., \& Jaureguizar, J. (2013). Conducta antisocial: 
conexión con emociones positivas y variables predictoras. Apuntes de Psicología, 31(2), 123-133. doi:10.1016/j.psi.2015.12.002

*(29) Garaigordobil, M., Machimbarrena, J., \& Maganto, C. (2016). Adaptación española de un instrumento para evaluar la resolución de conflictos (Conflictalk): Datos psicométricos de fiabilidad y validez. Revista de Psicología Clínica con Niños y Adolescentes, 3(2), 59-67.

*(30) García-Sancho, E., Salguero, J., \& Fernández-Berrocal, P. (2017). Ability emotional intelligence and its relation to aggression across time and age groups. Scandinavian Journal of Psychology, 58(1), 43-51. doi:10.1111/sjop.12331

*(31) Gázquez, J., Pérez-Fuentes, M., DíazHerrero, Á., García-Fernández, J., \& Inglés, C. (2015). Perfiles de inteligencia emocional y conducta social en adolescentes españoles. Psicología Conductual, 23(1), 141-160.

Gismero E. (2000). EHS, Escala de Habilidades Sociales. Madrid: TEA Publicaciones de Psicología Aplicada.

Goleman, D. (1995). Emotional intelligence. New York: Bantam Books.

*(32) Gómez-Baya, D., Mendoza, R., \& Paino, S. (2016). Perceived emotional intelligence as a predictor of depressive symptoms after a one year follow-up during adolescence. International Journal of Emotional Education, 8(1), 35-47. doi:10.1016/j.paid.2016.08.022

*(33) González-Cabrera, J., Pérez-Sancho, C., \& Calvete, E. (2016). Diseño y validación de la "Escala de Inteligencia Emocional en Internet" (EIEI) para adolescentes. Psicología Conductual, 24(1), 93-105.

*(34) Guerrero, C., Sosa-Correa, M., Zayas, A., \& Guil, R. (2017). Regulación emocional en jóvenes deportistas ante situaciones adversas en competición. International Journal of Developmental and Educational Psychology, 2(1), 373-384. doi:10.17060/ijodaep.2017.n1.v2.950

Halle, T., \& Darling-Churchill, K. (2016). Review of measures of social and emotional development. Journal of Applied Developmental Psychology, 45, 8-18. doi:10.1016/j.appdev.2016.02.003

Humphrey, N., Kalambouka, A., Wigelsworth, M., Lendrum, A., Deighton, J., \& Wolpert, M.
(2011). Measures of social and emotional skills for children and young people: A systematic review. Educational and Psychological Measurement, 71(4), 617-637. doi:10.1177/0013164410382896

*(35) Inglés, C., Torregrosa, M., GarcíaFernández, J., Martínez-Monteagudo, M., Estévez, E., \& Delgado, B. (2014). Conducta agresiva e inteligencia emocional en la adolescencia. European Journal of Education and Psychology, 7(1), 29-41.

doi:10.30552/ejep.v7i1.97

International Test Commission. (2017). The ITC Guidelines for Translating and Adapting Tests (Second edition).

*(36) Jiménez, M., \& López-Zafra, E. (2011). Social attitudes and social adaptation among Spanish adolescents: The role of perceived emotional intelligence. Revista de Psicología Social, 26(1), 105-117. doi:10.1174/021347411794078417

*(37) Martín, M., Guzmán, J., \& de Benito, A. (2018). Inteligencia emocional, percepción de apoyo a la autonomía y relaciones en el deporte. Cuadernos de Psicología del Deporte, 18(1), 13-20.

*(38) Mateu-Martínez, O., Piqueras, J., JiménezAlbiar, M., Espada, J., Carballo, J., \& Orgilés, M. (2013). Eficacia de un programa de prevención cognitivo-conductual breve del rechazo social en niños. Terapia psicológica, 31(2), 187-195. doi:10.4067/s0718-48082013000200005

Mayer, J., Caruso, D., \& Salovey, P. (2016). The ability model of emotional intelligence: Principles and updates. Emotion Review, 8(4), 290-300. doi:10.1177/1754073916639667

Mayer, J., Roberts, R., \& Barsade, S. (2008). Human abilities: Emotional intelligence. Annual Review of Psychology, 59, 507-536. doi:10.1146/annurev.psych.59.103006.093646

Mayer, J., \& Salovey, P. (1997). What is emotional intelligence? En P. Salovey y D. Sluyter (Eds.), Emotional development and emotional intelligence: Implications for educators (pp. 3-31). New York, NY: Basic Books.

Mayer, J., Salovey, P., \& Caruso, D. (2002). MayerSalovey-Caruso Emotional Intelligence Test 
(MSCEIT) item booklet, Version 2.0. Toronto, Ontario, Canada: MHS Publishers.

*(39) Mera, M., Martínez-Taboada, C., \& Elgorriaga, E. (2014). Rendimiento académico, ajuste escolar e inteligencia emocional en adolescentes inmigrantes y autóctonos. Boletín de Psicología, 110, 69-82. doi:10.13140/2.1.4491.5842

*(40) Mestre, J., Guil, R., Lopes, P., Salovey, P., \& Gil-Olarte, P. (2006). Emotional intelligence and social and academic adaptation to school. Psicothema, 18, 112117.

Mestre, J., Guil, R., Martínez-Cabañas, F., Larrán, C., \& González, G. (2011). Validación de una prueba para evaluar la capacidad de percibir, expresar y valorar emociones en niños de la etapa infantil. REIFOP, 14 (3), 37-57.

Moher, D., Liberati, A., Tetzlaff, J., \& Altman, D. (2010). Preferred reporting items for systematic reviews and meta-analyses: The PRISMA statement. International Journal of Surgery, 8(5), 336-341.

doi:10.7326/0003-4819-151-4-20090818000135

Nathanson, L., Rivers, S. E., Flynn, L. M., \& Brackett, M.A. (2016). Creating emotionally intelligent schools with RULER. Emotion Review, 8(4), 1-6.

doi:10.1177/1754073916650495er.sagepub.com

Omar, A., Salessi, S., Urteaga, F., \& Vaamonde, J. (2014). Validación transcultural de la Escala de Inteligencia Emocional de Schutte. Diversitas: Perspectivas en Psicología, 10(2), 261-274.

*(41) Passos, E. (2014). Características emocionales, familiares y sociales presentes en los niños, niñas y adolescentes en riesgo de explotación laboral. Revista Tendencias y Retos, 19(2), 45-61.

*(42) Pedregosa, P. (2017). La inteligencia emocional en el contexto educativo de adolescentes cordobeses. Revista de Estudios e Investigación en Psicología y Educación, (05), 005-008.

doi:10.17979/reipe.2017.0.05.2138

*(43) Pena, M., Extremera, N., \& Rey, L. (2011). El papel de la inteligencia emocional percibida en la resolución de problemas sociales en estudiantes adolescentes. Revista
Española de Orientación y Psicopedagogía, 22(1), 69-79.

doi:10.5944/reop.vol.22.num.1.2011.78

*(44) Pérez, N., \& Alegre, A. (2012). La Inteligencia y la seguridad emocional: El caso de la adaptación de los preadolescentes en la escuela. Revista Iberoamericana de Educación, 60(1), p. 1-11.

Petrides, K. (2012). Technical manual for the trait emotional intelligence questionnaire (TEIQue). London: London Psychometric Laboratory.

Petrides, K., \& Furnham, A. (2001). Trait emotional intelligence: Psychometric investigation with reference to established trait taxonomies. European Journal of Personality, 15(6), 425-448. doi:10.1002/per.416

*(45) Pulido, F. (2017). Diferencias por cultura en una validación inicial de un instrumento en construcción para evaluar la inteligencia emocional en una muestra de alumnos de primaria. International Journal of Developmental and Educational Psychology, 3(1), 173-182. doi:10.17060/ijodaep.2017.n1.v3.986

*(46) Pulido, F., \& Herrera, F. (2017). Predictores de la ansiedad y la inteligencia emocional en la adolescencia. Apuntes de Psicología, 35(1), 63-71.

*(47) Pulido, F., \& Herrera, F.(2015). Miedo, inteligencia emocional y rendimiento académico adolescente en el contexto pluricultural de Ceuta. Revista de Psicología GEPU, 6(2), 31.-52.

*(48) Ramírez-Lucas, A., Ferrando, M., \& Sáinz, A. (2015). ¿Influyen los estilos parentales y la inteligencia emocional de los padres en el desarrollo emocional de sus hijos escolarizados en $2^{\circ}$ Ciclo de educación infantil? Acción Psicológica, 12(1), 65-78 doi:10.5944/ap.12.1.14314

*(49) Ramos-Díaz, E., Jiménez-Jiménez, V., Rodríguez-Fernández, A., Fernández-Zabala, A., \& Axpe, I. (2017). Entrenamiento de la inteligencia emocional en el caso de una adolescente víctima de maltrato infantil. Revista de Psicología Clínica con Niños y Adolescentes, 4(1), 17-24. 
*(50) Reina, M., \& Oliva, A. (2015). De la competencia emocional a la autoestima y satisfacción vital en adolescentes. Psicología Conductual, 23(2), 345-359.

Resurrección, D., Salguero, J., \& Ruiz-Aranda, D. (2014). Emotional intelligence and psychological maladjustment in adolescence:

A systematic review. Journal of adolescence, 37(4), 461-472.

doi:10.1016/j.adolescence.2014.03.012

*(51) Rey, L., \& Extremera, N. (2012). Inteligencia emocional percibida, felicidad y estrategias distractoras en adolescentes. Boletín de psicología, 104, 87-101.

*(52) Rey, L., Extremera, N., \& Pena, M. (2011). Perceived emotional intelligence, self-esteem and life satisfaction in adolescents. Psychosocial Intervention, 20(2), 227-234. doi:10.5093/in2011v20n2a10

Rieffe, C., Oosterveld, P., Miers, A., Terwogt, M., \& Ly, V. (2008). Emotion awareness and internalising symptoms in children and adolescents: The Emotion Awareness Questionnaire revised. Personality and Individual Differences, 45, 756-761. doi:10.1 016/j.paid. 2008.08.001

Rockhill, C., \& Greener, S. (1999). Development of the Trait Meta-Mood Scale for elementary school children. Póster presentado en el Biennial Meeting of the Society for Research in Child Development.Alburquerque, NM.

Rodrigo-Ruiz, D., Cejudo, J., \& Pérez-González, J. (2019). Compendio y análisis de medidas de evaluación de la inteligencia emocional capacidad. Revista iberoamericana de diagnóstico y evaluación psicológica, 2(51), 99-116. doi:10.21865/RIDEP51.2.08

*(53) Roque, J. (2012). La inteligencia emocional en adolescentes del segundo curso de secundaria de la Unidad Educativa "Germán Busch". Revista de Investigación Psicológica, (8), 57-73.

Ruiz-Aranda, D., Cabello, R., Salguero, J., Extremera, N., \& Fernández-Berrocal, P. (2013). Programa Intemo. Guía para mejorar la inteligencia emocional de los adolescentes. Madrid: Pirámide.

*(54) Ruiz-Aranda, D., Fernández-Berrocal, P., Cabello, R., \& Extremera, N. (2006). Inteligencia emocional percibida y consumo de tabaco y alcohol en adolescentes. Ansiedad y Estrés, 12, 223-230.

*(55) Ruvalcaba, N., Gallegos, J., Borges, A., \& Gonzalez, N. (2017). Extracurricular activities and group belonging as a protective factor in adolescence. Psicología Educativa, 23(1), 4551. doi:10.1016/j.pse.2016.09.001

Ruvalcaba, N., Gallegos, J., Lorenzo, M., \& Borges, A. (2014). Propiedades psicométricas del inventario de competencias socioemocionales para adolescentes (EQIYV) en población mexicana. Revista Evaluar, 14(1), 1-14.

*(56) Ruvalcaba, N., Murrieta-Cummingsy, P., \& Arteaga-Velázquez, A. (2016). Competencias socioemocionales y percepción de la comunidad ante la conducta agresiva en adolescentes que viven en entornos de riesgo. Acción Psicológica, 13(2), 79-88.

doi:10.5944/ap.13.2.17816

*(57) Sainz, M., Bermejo, R., Ferrándiz, C., Prieto, M., \& Ruiz, M. (2015). Cómo funcionan las competencias socioemocionales en los estudiantes de alta habilidad. Aula, 21, 33.-47. doi:10.14201/aula2015213347

Sáinz, M. Ferrándiz, C., Fernández, M., \& Ferrando, M. (2014) Propiedades psicométricas del Inventario de Cociente Emocional EQ-i:YV en alumnos superdotados y talentosos. Revista de Investigación Educativa, 32 (1), 41-55. doi:10.6018/rie.32.1.162501

*(58) Salavera, C., Usán, P., \& Jarie, L. (2017). Emotional intelligence and social skills on self-efficacy in Secondary Education students. Are there gender differences? Journal of adolescence, 60, 39-46

doi:10.1016/j.adolescence.2017.07.009

Salguero, J., Fernández-Berrocal, P., Balluerka, N., \& Aritzeta, A. (2010). Measuring perceived emotional intelligence in the adolescent population: Psychometric properties of the Trait Meta Mood Scale. Social Behavior and Personality, 38, 11971210. doi:10.2224/sbp.2010.38.9.1197

Salovey, P., Mayer, J., Goldman, S., Turvey, C., \& Palfai, T. (1995). Emotional attention, clarity, and repair: Exploring emotional intelligence using the Trait Meta-Mood Scale. En J. Pennebaker (Ed.), Emotion, Disclosure 
and Health (pp.125-151). Washington, DC: American Psychological Association.

*(59) Samper-García, P., Mesurado, B., Richaud, M., \& Llorca, A. (2016). Validación del Cuestionario de Conciencia Emocional en Adolescentes Españoles. Interdisciplinaria, 33(1), 163-176.

doi:10.16888/interd.2016.33.1.10

Sánchez-Álvarez, N., Extremera, N., \& Fernández-Berrocal, P. (2016). The relation between emotional intelligence and subjective well-being: A meta-analytic investigation. The Journal of Positive Psychology, 11(3), 276285. doi:10.1080/17439760.2015.1058968

*(60) Santamaría, B., \& Valdés, M. (2017). Rendimiento del alumnado de educación secundaria obligatoria: Influencia de las habilidades sociales y la inteligencia emocional. International Journal of Developmental and Educational Psychology, 2(1), 57-66. doi:10.17060/ijodaep.2017.n1.v2.918

*(61) Serrano, C., \& Vaillo, Y. (2016). Inteligencia emocional percibida, bienestar subjetivo, estrés percibido, engagement y rendimiento académico en adolescentes. Revista de Psicodidáctica, 21(2), 357-374. doi:10.1387/RevPsicodidact.14887

Schutte, N., Malouff, J., Hall, L., Haggerty, D., Cooper, J., Golden, C., \& Dornheim, L. (1998). Development and validation of a measure of emotional intelligence. Personality and Individual Differences, 25(2), 167-177. doi:10.1016/S0191-8869(98)000014

*(62) Ugarriza, N., \& Pajares, L. (2005). La evaluación de la inteligencia emocional a través del inventario de BarOn ICE: NA, en una muestra de niños y adolescentes. Persona, (8), 11-58. doi:10.26439/persona2005.n008.893

*(63) Valadez, M., Pérez, L., \& Beltrán, J. (2010). La inteligencia emocional de los adolescentes talentosos. Faisca: Revista de Altas Capacidades, 15(17), 2-17.

*(64) Verdugo, E., Arias, M., Martínez, R., Jalón, M., \& Díaz-Aguado, J. (2010). Sexismo, inteligencia emocional y adolescencia. Psicología Educativa, 16(1), 77-88. doi:10.5093/ed2010v16n1a7
*(65) Villanueva, L., Montoya-Castilla, \& PradoGasco, V. (2017). The importance of trait emotional intelligence and feelings in the prediction of perceived and biological stress in adolescents: Hierarchical regressions and fsQCA models. Stress, 20(4), 355-362 doi:10.1080/10253890.2017.1340451

Wigelsworth, M., Humphrey, N., Kalambouka, A., \& Lendrum, A. (2010). A review of key issues in the measurement of children's social and emotional skills. Educational Psychology in Practice, 26(2), 173-186. doi:10.1080/02667361003768526

Wong, C., \& Law, K. (2002). The effects of leader and follower emotional intelligence on performance and attitude: An exploratory study. Leadersh, 13, 243-274. doi:10.1016/s1048-9843(02)00099-1

*(66) Zavala, M., Valadez, M., \& Vargas, M. (2008). Inteligencia emocional y habilidades sociales en adolescentes con alta aceptación social. Electronic Journal of research in educational Psychology, 6(15), 321-338. doi:10.25115/ejrep.v6i15.1291

*(67) Zavala, M., \& López, I. (2012). Adolescentes en situación de riesgo psicosocial:¿Qué papel juega la inteligencia emocional? Psicología Conductual, 20(1), 5975.

Zeman, J., Klimes-Dougan, B., Cassano, M., \& Adrian, M. (2007). Measurement issues in emotion research with children and adolescents. Clinical Psychology: Science and Practice, 14(4), 377-401.

*(68) Zych, I., Ortega-Ruiz, R., \& Marín-López, I. (2017). Emotional content in cyberspace: Development and validation of E-motions Questionnaire in adolescents and young people. Psicothema, 29(4), 563-569 doi:10.7334/psicothema2016.340 